\title{
CORPUS Corpus
}

15 | 2016

Corpus de français parlé et français parlé des corpus

\section{Corpus international écologique de la langue française (CIEL-F) : un corpus pour la recherche comparée sur le français parlé}

The international ecological linguistic corpus of French (CIEL_F): A database for comparative research in spoken French

\section{Lorenza Mondada et Stefan Pfänder}

\section{OpenEdition \\ Journals}

Édition électronique

URL : http://journals.openedition.org/corpus/2951

ISSN : 1765-3126

Éditeur

Bases; corpus et langage - UMR 6039

Édition imprimée

Date de publication : 15 octobre 2016

ISSN : 1638-9808

Référence électronique

Lorenza Mondada et Stefan Pfänder, « Corpus international écologique de la lanque française (CIELF) : un corpus pour la recherche comparée sur le français parlé ", Corpus [En ligne], 15 | 2016, mis en ligne le 15 janvier 2017, consulté le 08 septembre 2020. URL : http://journals.openedition.org/corpus/ 2951

Ce document a été généré automatiquement le 8 septembre 2020

(C) Tous droits réservés 


\section{Corpus international écologique de la langue française (CIEL-F) : un corpus pour la recherche comparée sur le français parlé}

The international ecological linguistic corpus of French (CIEL_F): A database for comparative research in spoken French

Lorenza Mondada et Stefan Pfänder

\section{Introduction}

1 Cet article a pour objectif de présenter le travail de constitution du Corpus international écologique de la langue française (CIEL-F) et quelques potentialités, mais aussi défis, qu'il implique pour une analyse comparée du français dans différentes zones communicatives et au sein de différents types d'activités. Pour ce faire, l'article est organisé en deux parties, la première présentant le corpus et le projet qui l'a motivé, et la seconde offrant un exemple d'analyse.

2 Il existe aujourd'hui plusieurs corpus de français oral disponibles, qui ont été conçus à partir d'arrière-plans théoriques et disciplinaires, impliquant par là nécessairement des méthodologies distinctes qui ont des effets sur la manière dont les données sont collectées et transcrites - notamment $\mathrm{PFC}^{1}$ conçu à partir d'un intérêt pour la phonologie du français, ESLO² à partir d'un projet sociolinguistique, poursuivi à travers deux phases de recueil historiquement bien distinctes (1968 et actuellement), CLAPI ${ }^{3}$ à partir des principes de la linguistique interactionnelle, VALIBEL ${ }^{4}$ à partir de la sociolinguistique variationniste, etc. Ces banques de données constituent des corpus d'une richesse importante qui rendent difficile de répéter aujourd'hui les constats sur le manque de données pour le français qui ont émaillé la littérature pendant longtemps. Toutefois, cette richesse de données laisse intacts plusieurs problèmes : les 
"données " y sont définies de façons très diverses, allant de tâches plus ou moins écologiques, plus ou moins dialogiques ou monologiques, aux interviews et aux interactions sociales situées; les enregistrements couvrent de manière très hétérogène les espaces régionaux, nationaux et internationaux où le français est parlé, laissant de nombreuses régions géolinguistiques dans l'ombre, et certains types d'activités y sont sur-représentés (p. ex. : l'entretien ou la conversation), alors que de nombreux autres sont alors plus ou moins totalement ignorés ; les métadonnées documentées sont très diverses; les transcriptions obéissent à des standards mais aussi à des niveaux de granularités très hétérogènes; les données sont plus ou moins annotées ou taguées, selon des conventions différentes; les corpus sont plus ou moins interrogeables, par des outils qui, là aussi, varient énormément dans les types de requêtes qu'ils permettent de formuler. Ce constat met aujourd'hui au centre de l'attention technologique la question de l'interopérabilité des corpus/des banques de données et au centre de l'attention analytique la question de la comparabilité des données.

3 Le corpus CIEL $^{5}$ a été constitué au sein d'un projet financé par le programme francoallemand ANR-DFG ${ }^{6}$, réunissant 5 partenaires : Françoise Gadet (UMR MoDyCO, Paris), Ralph Ludwig (Univ. Halle), Lorenza Mondada (UMR ICAR, Lyon), Stefan Pfänder (Univ. Freiburg-im-Breisgau), et Anne-Catherine Simon (Univ. Louvain-la-Neuve), pendant la période 2008-2012. Le projet répond à une partie des questions évoquées ci-dessus : émanant de linguistes actifs dans les domaines de la grammaire de l'oral, de la sociolinguistique et de la linguistique interactionnelle, il vise un corpus qui puisse permettre d'accéder à des données du français enregistrées dans des conditions écologiques dans différentes aires communicatives, recueillies dans des contextes comparables, renseignées par rapport aux mêmes métadonnées, transcrites avec les mêmes conventions et critères. Les corpus sont réunis dans une banque de données qui réunit les expertises des équipes ayant réalisé la plateforme $\mathrm{CLAPI}^{7}$ (Corpus de langue parlée en interaction) au laboratoire ICAR à Lyon et développé le système [moca] ${ }^{8}$ (multimodal oral corpora administration) à Freiburg et à Louvain-la-Neuve.

\section{Le corpus CIEL : critères de constitution}

Le corpus CIEL vise à rassembler des données de français recueillies en différents points géographiques sur plusieurs continents, enregistrées dans des conditions écologiques (voir Dister et al., 2008 ; Gadet, Ludwig, Mondada, Pfänder \& Simon, 2012).

5 Il s'agit tout d'abord d'un corpus écologique : ce terme renvoie à plusieurs acceptions dans la littérature que le projet CIEL tente d'intégrer et d'articuler (voir Ludwig, Mühlhäusler \& Pagel, éds, à paraitre). Haugen (1972) est parmi les premiers à utiliser ce terme en linguistique, renvoyant par là à une conception de la langue comme inscrite dans un réseau complexe d'interdépendances, que ce soit avec l'environnement social et naturel ou avec d'autres langues, et dont l'ancrage social se manifeste concrètement dans la communication en situation d'interaction. À partir de Haugen, l'écologie linguistique a pris d'une part au sérieux le concept biologique, en pensant l'évolution linguistique en termes de langue en train de survivre, de se développer ou bien de disparaître; d'autre part le concept a été utilisé de manière métaphorique pour renvoyer à un système d'interdépendances plus abstrait et aussi davantage orienté vers les dynamiques sociales. L'approche de l'écologie dans le projet CIEL distingue différents niveaux: le niveau macro-écologique reconnaît la formation historique de 
contextes d'usage du français - comme, par exemple, la francophonie africaine ou le Levant - ; le niveau méso-écologique tient compte des contextes urbains, régionaux, ou insulaires ( $c f$. infra, les aires communicatives) ; le niveau micro-écologique considère les contextes sociaux et institutionnels d'usage du français, ainsi que les environnements spatiaux et matériels dans lesquels la langue est utilisée comme ressource pour l'interaction sociale située ( $c f$. infra, les types d'activité). Méthodologiquement, la dimension écologique est comprise et implémentée dans le corpus CIEL sous la forme de l'exigence de se doter d'enregistrements de la parole située dans son contexte social ordinaire et routinier, sans qu'elle soit élicitée ou orchestrée par le chercheur (Mondada, 2012).

6 Ces différents principes issus de la notion d'écologie ont été traduits dans le corpus CIEL sous la forme d'une triple exigence : des données naturalistes enregistrées in situ qui documentent une variété de zones communicatives - qui émanent d'une typologie des aires - et des types d'activités distincts - qui émanent d'une typologie des situations d'usage.

7 La typologie des aires communicatives (cf. Gadet, Ludwig \& Pfänder 2009) est inspirée d'une révision critique de la question de l'ancrage spatial, géographique et politique des aires linguistiques. L'aire communicative renvoie à un espace qui est moins défini de manière géopolitique ou selon les frontières de l'État-nation, que dans la perspective des locuteurs et de leurs usages, faisant intervenir le prestige, la vitalité, le contact avec d'autres variétés linguistiques, ainsi que la fonctionnalité communicative. La typologie se fonde sur quatre critères: a) l'espace socio-géographique (distinguant entre centres urbains et zones plus périphériques et rurales), b) le contact linguistique (distinguant entre formes de contact dominantes vs non-dominantes, avec une vs plusieurs variétés ; entre contact avec une langue typologiquement proche (européenne) ou non; et entre contact avec des variétés écrites vs non écrites), c) la dimension fonctionnelle (contemplant les types d'usages, dans différents contextes, formels vs informels, privés vs publics, en situation d'oralité vs de littératie) et d) la dimension de dynamisme langagier (incluant la vitalité vs l'obsolescence de la variété considérée). Sur cette base, différentes aires en Algérie, Antilles françaises, Belgique, Burkina Faso, Cameroun, Canada, Congo, Côte d'Ivoire, Égypte, France, Inde, La Réunion, Maurice, Sénégal, Suisse et Togo ont été documentées.

8 La typologie des activités communicatives a été privilégiée par rapport à un échantillonnage des locuteurs qui régit souvent les enquêtes sociolinguistiques, mais qui soulève de nombreux problèmes dès que l'on pense moins en termes d'individus et davantage en termes d'interactions sociales. Ces activités sont abordées en respectant leur caractère situé : il ne s'agit pas d'activités provoquées ou orchestrées par un enquêteur (cela exclut les entretiens, les données sollicitées ou expérimentales), mais d'activités telles qu'elles se déroulent ordinairement (Mondada, 2012; Groupe ICOR, 2010). Le choix des activités s'inspire des travaux de l'ethnographie de la communication, de la linguistique anthropologique et de l'analyse conversationnelle. En particulier, il a été inspiré par la notion de «speech event » de Gumperz (1982) et d'«activity type " de Levinson (1979). Les activités ainsi définies sont à la fois solidement ancrées dans leur contexte et repérables dans des contextes divers. Le corpus a privilégié des types d'activités répondant à une série de critères sociointeractionnels : a) des échanges interactionnels comportant plus de deux et si possible moins de six locuteurs (afin de limiter le risque de schismes conversationnels), 
b) constituant des activités dont les frontières temporelles et spatiales sont naturellement identifiées par les locuteurs (comme un repas ou une réunion, délimitées entre un début et une fin vers lesquelles s'orientent les participants), c) ainsi que des activités reconnaissables de manière endogène (émique) par les participants et membres du groupe concerné, d) qu'il est possible de documenter, avec des variations, dans des cultures et des sociétés très différentes, e) et qui permettent d'observer des pratiques langagières et interactionnelles diversifiées (contextes ordinaires informels vs institutionnels, contextes faisant intervenir des groupes de même âge vs différentes générations, interactions plus ou moins ancrées dans le corps et la gestualité, pouvant aussi intéresser l'analyse multimodale). Du point de vue méthodologique, il s'agissait aussi de retenir des types d'activités accessibles à l'enregistrement, ne posant pas de problèmes de confidentialité, et, du point de vue éthique et juridique, pouvant être diffusés (cela motivant l'exclusion de situations impliquant des contenus confidentiels et intimes). Sur cette base, trois types d'activités ont été privilégiés :

- conversations durant des repas entre amis ou en famille ;

- interactions en contexte professionnel (par exemple réunions);

- interactions enregistrées sur une radio locale.

9 Pour chaque aire et chaque type d'activité, 3 enregistrements ont été visés - avec actuellement un corpus constitué de 183 enregistrements.

\section{Traitement et archivage des corpus : des données aux banques de données}

10 Le travail effectué pour réaliser le corpus CIEL implique de nombreux paliers et un réseau de collaborations et d'expertises. Le corpus a été conçu par les 5 équipes en charge du projet. Les données ont été enregistrées en partie par les mêmes équipes en partie par des collaborateurs distribués sur plusieurs continents et qui ont accepté de contribuer au projet. Elles ont été ensuite transcrites, en grande partie par les équipes du projet mais aussi avec l'aide des collaborateurs externes ${ }^{9}$. Enfin, elles ont été alignées, décrites par des métadonnées et intégrées sous un format XML à la base de données. Chacune de ces étapes suppose un travail de coordination et de standardisation important, qui pose des problèmes conceptuels, méthodologiques et techniques, mais aussi disciplinaires, épistémologiques et humains.

11 Le travail d'enregistrement des données a souvent reposé sur un contact étroit avec les locuteurs sur place et donc sur la collaboration avec des personnes y résidant ou y travaillant. Cela a supposé une réflexion en matière de conception des enregistrements et de recours à des technologies adéquates, implémentée dans un protocole pour le recueil d'enregistrements audio et vidéo (Mondada, 2011). Cela a supposé surtout la formation correspondante des équipes et la résolution de nombreux problèmes techniques, notamment dans les zones les plus périphériques et lointaines, ne disposant pas des mêmes équipements. Les conditions d'accès au terrain sont très différentes d'un contexte culturel à un autre : selon les sites, le fait même d'enregistrer est largement accepté voire banalisé, par les usages de Facebook et par une acceptation tacite de la vidéo-surveillance, ou bien il est rejeté, du fait d'une sensibilisation à la surmédiatisation des enregistrements de la vie privée et aux violations de l'intimité des personnes, ou encore il fait l'objet de résistances plus ou moins ouvertes, du fait de conceptions très différentes de ce qu'est la sphère "privée ». De même, les possibilités 
d'enregistrer peuvent fortement varier d'un contexte national à l'autre : par exemple, dans certains contextes académiques, il est déontologiquement et juridiquement difficile pour les chercheurs d'enregistrer des conversations privées, les interactions institutionnelles étant plus accessibles (Canada), alors que dans d'autres ce sont les interactions institutionnelles et professionnelles qui posent des problèmes de confidentialité (Europe).

Le travail de transcription a également reposé sur un travail important d'harmonisation et de standardisation des pratiques et des conventions. Comme on le sait, la transcription repose sur une série de choix qui explicitement ou tacitement reposent sur des modèles et des présupposés théoriques (Ochs, 1979), distinguant souvent des communautés disciplinaires mais aussi, au sein de mêmes traditions épistémologiques, nationales. La démarche d'harmonisation et de standardisation passe donc par des négociations qui sont aussi bien théoriques que pratiques. C'est ainsi qu'une convention a été préalablement discutée entre les équipes du projet (Simon, Gadet, Ludwig, Mondada, Pfänder \& Skrovec, 2012) sur la base des bonnes pratiques existantes. Acceptant le caractère fini, sélectif et interprétatif de la transcription comme donnée secondaire - dont le caractère contraignant est relativisé par la possibilité de la vérifier sur la base de l'enregistrement ou donnée primaire -, le projet CIEL a procédé à une transcription fondée sur un certain nombre de principes de base. Parmi eux, citons l'adoption d'une transcription orthographique standard pour faciliter la lisibilité mais aussi l'interrogeabilité des textes, doublée d'une transcription orthographique adaptée pour s'approcher davantage de la production orale enregistrée, ainsi que d'une traduction ou glose pour les passages qui le nécessitaient (dans d'autres langues que le français ou dans des variétés particulières). Ces différentes versions sont toutes accessibles sous le logiciel Praat ${ }^{10}$, qui permet un alignement entre le signal sonore et les différentes lignes (tiers) de la transcription: cela facilite non seulement l'analyse prosodique, mais surtout permet la multiplication virtuellement infinie des lignes d'annotation pour différentes finalités; en outre ce format (text-grid) est exportable à la fois comme texte et comme fichier intégrable dans une banque de données. De même, un script Praat a été utilisé pour l'anonymisation (par beepage permettant de garder le même contour prosodique du segment anonymisé) des détails personnels privés évoqués par des participants dans les enregistrements.

Chaque enregistrement et sa description ont fait l'objet d'une description sous forme de métadonnées, dont les champs à renseigner ont aussi fait l'objet préalable de discussions et négociations auprès des équipes du projet. Le choix des catégories à renseigner implique non seulement des intérêts de recherche mais aussi et d'abord des présupposés théoriques. Par exemple, loin d'être un simple recueil d'informations objectives sur les locuteurs, les métadonnées révèlent des choix théoriques concernant les dimensions pertinentes pour définir l'identité (culturelle, sociale, linguistique...) du locuteur. Ainsi consigner les langues parlées par le locuteur amène à une discussion sur le statut de catégories telles que "locuteur natif», "langue première ", "langue maternelle», "langue seconde» etc.; consigner son appartenance socio-économique soulève la question de la segmentation et description pertinentes de la structuration sociale d'un groupe; consigner son appartenance ethnique soulève des problèmes épineux de catégorisation. Ces problèmes ont été discutés dans la littérature (voir par exemple la membership categorisation analysis de Sacks, 1972), mais l'implémentation de ces discussions dans des choix au sein d'une série de métadonnées, elles-mêmes à 
traduire dans des questionnaires à soumettre sur le terrain, n'est pas un processus linéaire et évident. En outre, les métadonnées ne concernent pas uniquement les locuteurs et les phénomènes enregistrés, mais documentent également les conditions d'enregistrement et de transcription. Ces renseignements sont une trace importante d'un processus réflexif dont certains détails peuvent se révéler importants lors des analyses. Cela ne fait qu'exhiber la différence radicale entre les corpus que les chercheurs enregistrent pour leurs propres analyses et les corpus que des chercheurs recueillent pour les mettre à disposition de la communauté. Dans ce dernier cas, l'architecture du corpus, et crucialement les métadonnées, construisent la possibilité, qui ne va pas de soi, que l'on puisse procéder à une analyse de données que l'on n'a pas soi-même collectées.

14 Les enregistrements, les transcriptions et les métadonnées ont été ensuite consignés dans une architecture informatique en permettant l'archivage ainsi que l'interrogation, sous la forme d'une banque de données. Celle-ci est accessible à partir de deux interfaces, liées à deux plateformes : CLAPI et [moca].

15 CLAPI est une banque de données de corpus de parole en interaction enregistrées en audio et en vidéo, développée depuis les années 2000 au laboratoire ICAR à Lyon et mettant actuellement librement à disposition de la communauté scientifique 50 heures de données interrogeables, 120 corpus transcrits, 30 heures de données téléchargeables ${ }^{11}$. CLAPI peut être utilisé comme une archive de corpus, permettant d'en identifier, sélectionner et consulter les données primaires et les données secondaires, alignées sur le même timing, sur la base d'un riche jeu de métadonnées, mais fonctionne surtout comme une banque de données permettant de formuler des requêtes complexes, qui ont la particularité d'intégrer, au-delà des concordanciers habituels, des requêtes combinant recherche de formes linguistiques et de caractéristiques interactionnelles (par exemple : rechercher les occurrences de " non " en début de tour, éventuellement précédées de «euh» et de pauses inter tours et suivies d'un chevauchement par le tour de parole suivant) (voir Bert, Bruxelles, Étienne, Jouin-Chardon, Lascar \& Mondada, 2010, pour une présentation). Les requêtes effectuées et leurs résultats peuvent être sauvegardés dans un espace de travail personnalisé de l'usager.

[moca] est une plateforme ${ }^{12}$ qui permet d'administrer des corpus sous forme de fichiers audio et vidéo, et d'importer des fichiers txt/doc, ainsi que Exmaralda, Praat ou ELAN. Les corpus peuvent être recherchés et identifiés sur la base de métadonnées contenant la caractérisation sociolinguistique des locuteurs et les conditions de recueil du corpus. Des requêtes basées sur des suites de caractères permettent d'interroger les transcriptions ; les résultats sont affichés sous la forme de fragments de transcriptions dont le signal est consultable. La spécificité de cet outil est la visualisation des corpus sur une représentation cartographique, permettant de capturer en un coup d'œil la distribution géographique des données; il permet des recherches complexes et offre aussi la possibilité d'une annotation personnalisée des données par des labels et des étiquettes que l'usager peut créer et qu'il peut ensuite regrouper et sauvegarder sous la forme de sous-corpus (voir Ehmer \& Martinez, 2014, pour une présentation).

Le projet CIEL a fait le choix de permettre la consultation de ses données par le biais de ces deux interfaces: cela présente l'avantage de multiplier les possibilités quant à l'interrogation des données par des outils; de profiter de différentes interfaces selon les préférences des usagers; et d'exploiter les caractéristiques spécifiques des deux 
plateformes - notamment les requêtes complexes orientées vers la linguistique interactionnelle de CLAPI, les possibilités d'annotations personnalisées de [moca]. Une des conséquences de ce double choix est aussi une avancée dans la discussion aujourd'hui fondamentale - sur l'interopérabilité entre bases de données, grâce aux efforts conjoints des informaticiens des deux équipes (Carole Étienne à Lyon et Daniel Alcón à Freiburg).

\section{Enjeux analytiques}

La mise à disposition de corpus est un desideratum fort de la communauté scientifique ; leur mise à disposition avec des outils de requête et d'interrogation potentialise ces corpus et permet des analyses sur de grandes masses de données ; leur structuration dès la collecte - en un ensemble cohérent permettant différents types de comparaisons représente un apport encore plus crucial. Sur ce dernier point, l'originalité et la valeur du corpus CIEL-F est de mettre à disposition des données interrogeables de manière outillée qui sont fondées sur une conception du terrain, des enregistrements, des métadonnées et de l'architecture de la base de données, elle-même fondée sur la comparaison. CIEL-F permet la comparaison au sein de la même activité communicative, mais aussi entre activités communicatives (p. ex. : est-ce que voilà ou donc sont utilisés de la même manière dans des conversations ordinaires et des interactions professionnelles? Apparemment cela n'est pas le cas... Groupe ICOR, 2009, Pfänder \& Skrovec, 2011), au sein de la même aire communicative mais aussi, et surtout, entre aires communicatives. Ainsi, on peut se demander si une particule ou une construction grammaticale donnée est utilisée de la même manière dans des aires communicatives différentes (voir Skrovec \& Pfänder, 2012). La comparaison outillée est un des domaines les plus promettants de la recherche sur grands corpus - permettant d'envisager de revisiter des hypothèses passées non suffisamment documentées empiriquement ainsi que de formuler de nouvelles hypothèses et surtout de nouvelles analyses empiriques, au-delà de ce que l'on a coutume de dire ou de croire concernant, par exemple, la spécificité des variétés de français en Afrique ou la différence entre français canadien et français métropolitain.

Dans ce qui suit, nous n'avons pas la prétention d'épuiser une analyse comparative en quelques pages. Nous n'offrons que quelques pistes d'une analyse basée exclusivement sur des données extraites de CIEL-F donnant un exemple de phénomènes et de questions qu'il est possible d'envisager. L'exemple portera sur la forme là : elle présente l'intérêt d'avoir été déjà étudiée dans une littérature substantielle et d'avoir été utilisée pour illustrer la spécificité et les différences entre variétés du français (Italia, 2006, sur là en français du Gabon; Ludwig \& Pfander, 2003, sur là en créole caribéen et en français ; Ploog, 2006, sur là en français d'Abidjan, Queffélec et al., 1997, sur le français en Centrafrique ; Ngamountsika, 2012, sur là en français du Congo ; Wiesmath, 2003, sur là en acadien, Forget, 1989, Vincent, 1981, sur là en français du Canada - sans oublier les travaux sur le français métropolitain, notamment de Barbéris, 1992) -, mais sans avoir pu comparer directement le comportement de la forme dans des aires différentes.

Dans ce qui suit, nous nous penchons sur quelques variations, différences mais aussi similarités que l'on peut repérer à propos des emplois de là dans le corpus CIEL-F. 


\section{Là : des usages locatifs à la grammaticalisation en particule}

21 L'intérêt pour l'analyse d'une forme comme là est représenté par le fait qu'il est possible de la trouver dans toutes les positions envisageables au fil des corpus: au début, au milieu et à la fin de clauses comme de tours; après des noms, des verbes, des connecteurs et autres catégories; sous la forme d'une seule occurrence ou de deux occurrences répétées... La description fonctionnelle et catégorielle de la forme là correspond à cette richesse positionnelle. La multiplicité des rôles de là a été amplement soulignée par la littérature : là n'est pas uniquement un déictique mais a subi une grammaticalisation en marqueur discursif (aussi appelé là de clôture, Barbéris, 1992). Ainsi, par exemple, la typologie proposée par Ludwig \& Pfänder (2003 : 271-275 visant le français et le créole) identifie 5 types de là : spatial, démonstratif, situativotemporel, de structuration du discours antéposé et postposé. Ces 5 types, documentés dans le corpus CIEL-F, montrent qu'il existe un continuum allant d'emplois plus clairement spatiaux (c'est là, je vais là, ça et là) à des emplois où là perd son sens spatial pour devenir une particule discursive (l'homme là qui est venu là tu le connais là) - en passant par des emplois où le locatif devient davantage métaphorique et renvoie à un positionnement temporel (arrête là) ou dans l'espace-temps du discours (qu'est-ce que vous dites là ? je termine là).

Si l'on se penche sur le corpus CIEL et qu'on y recherche la forme là dans les différents types d'activité et dans la pluralité des aires communicatives documentées, on constate que ces emplois coexistent dans le temps et dans l'espace: dans un même fragment d'interaction, on peut trouver des occurrences de là avec un sens premièrement spatial et avec une fonction de structuration du discours. D'une part, à certains usages locatifs peuvent se superposer des usages interactionnels - comme le montre l'usage de là déictique avec un geste de pointage au service de l'auto-sélection (Mondada, 2007), au service de la structuration de l'activité (Mondada, 2014) ou au service du renforcement de la pertinence conditionnelle de la question (Mondada, à paraitre). D'autre part, la coexistence d'usages locatifs et d'usages de là grammaticalisé en particule peut amener les locuteurs - s'orientant ainsi vers la grammaticalisation comme perte du sens sémantique original - à adopter des stratégies de différenciation des deux types de ressources pour continuer à exprimer la dimension spatiale de là.

Nous mentionnons ici trois faits qui montrent cette double orientation dans les différentes aires du corpus.

La première évidence concerne l'occurrence de deux là successifs, comme dans les exemples suivants :

(1) (CA_NB_REP_04)

((en début d'enregistrement))

KAT: on va commencer/LÀ là \

(2) (CA_QC_REP_01)

A: on dirait qu-ils veulent pas qu'on

conteste rien parce que il a été éLU-

mais: tu sais ça s'arrête pas là là/

(3) (BE_WBR_REP_02)

PHI: ben: ils: rentrent chez eux/ hein tous

les gens: qui ont un: certain âge/

mais oui mais là LÀ c'était vraiment 
JEA: hm

PHI: c'était quasi pousser les gens dehors/ quoi

(4) (CI_ABJ_REP_02)

TAN: [c'est quoi c'est quoi qui est] écrit

là là/

(0.9)

MAB: quoi/

TAN: mou

(5) (FR_MZM)

COR: là là c'est un projet on va dire

c'est pour nous tesTER hein ça va être

pour voir la qualiTÉ: euh: pour voir

les délais: euh $\backslash$ (.)

ALI: d'a[ccord] où là-bas est positionné à la fin du tour ou de l'unité de construction du tour, position souvent occupée par le là de structuration (cf. infra § 6.) :

(6) (TP-P-PRO-01)

LOC: donc il va faire le feu là-bas

$(0.5)$

LOC: comme ça y aura la chaleur-

la fumée: qui va chasser les- ces

petits insectes-là

(7) (SN_DK_REP_01)

A: [il est ici//]

B: [cela est sûr $\backslash$ lui aussi:/ il est

(en train) de célébrer

le mariage là-bas

(8) (CI_ABJ_REP_02)

A: la semaine (0.4) dernière hein/ dix

jours aujourd'hui (0.4)

il y a eu un crash là-bas

(1.0)

A: et puis [aujourd'hui [encore y a y a xxxx

(9) (CM_DLA_REP-01)

A: c'est elle qui venait là-bas chaque

matin/ là

Dans le dernier exemple, là-bas est utilisé au sein du tour pour la référence spatiale, alors que là est utilisé à la fin du tour comme particule.

La troisième évidence est offerte par l'analyse multimodale - exploitant cette fois le fait que le corpus CIEL-F comprend aussi quelques données vidéo (bien qu'encore trop peu). Ainsi, dans l'extrait suivant, différentes formes de là sont utilisées. Le locuteur les 
différencie en utilisant ou non des gestes l'accompagnant - ce qu'il fait pour le là spatial, alors qu'il ne le fait pas pour le là grammaticalisé en particule :

(10) FR_LIO

1 ROG: h parce que tu vois moi y en a un

2 ou deux/ qui m'ont demanDÉ/ euh: sur

3 les questions de réglementation:/ (0.3)

4 .h d'avoir aussi/ (.) euh:: les textes/

5 et des fois la discussion autour du

6 texte/ et la compréhension du texte

$7(0.3)$

8 ROG: :h

9 BER: [ouais]

10 ROG: [et je] me dis que le- du coup le

$11 \rightarrow$ petit: texte *là \#(0.4) h$^{*}$ qu'on a

*paume latérale*

fig \#fig. 1

12 mis dans la pochette/ l'arrêté du

13 vingt-deux juin avec rénovation $B A F A$

14 B A F D/ (0.3) .h au MOINS repren-

15 en reprenant la partie animateur

Fig. 1

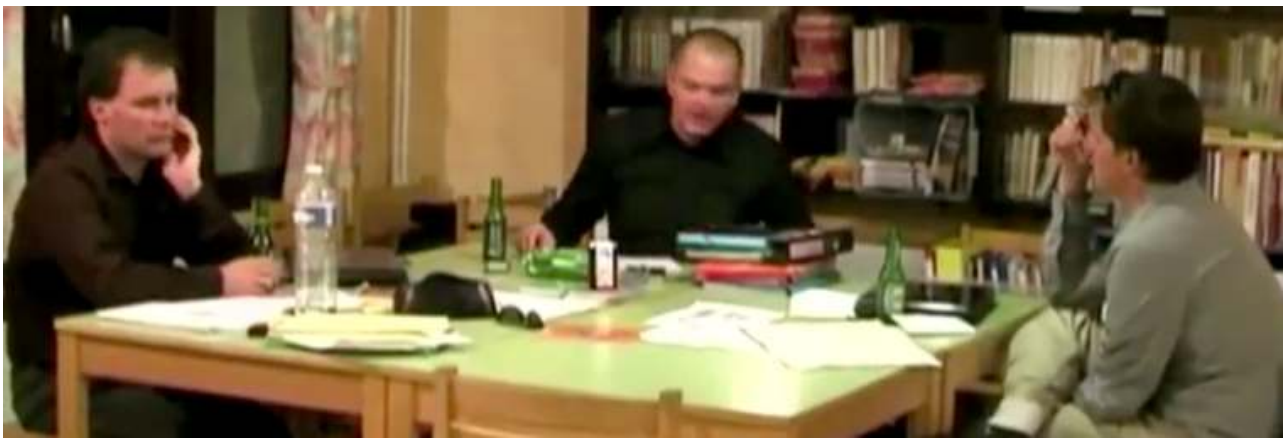

16 euh::(0.4) .h [euh:m:]

17 BER: [ils l'ont/ la]

18 partie [animateur/

19 ROG: [ouais ouais] ouais y- je

$20 \rightarrow$ leur ai mis [la com]plète là\#

fig \#fig.2

21 BER: [d'accord] 
Fig. 2

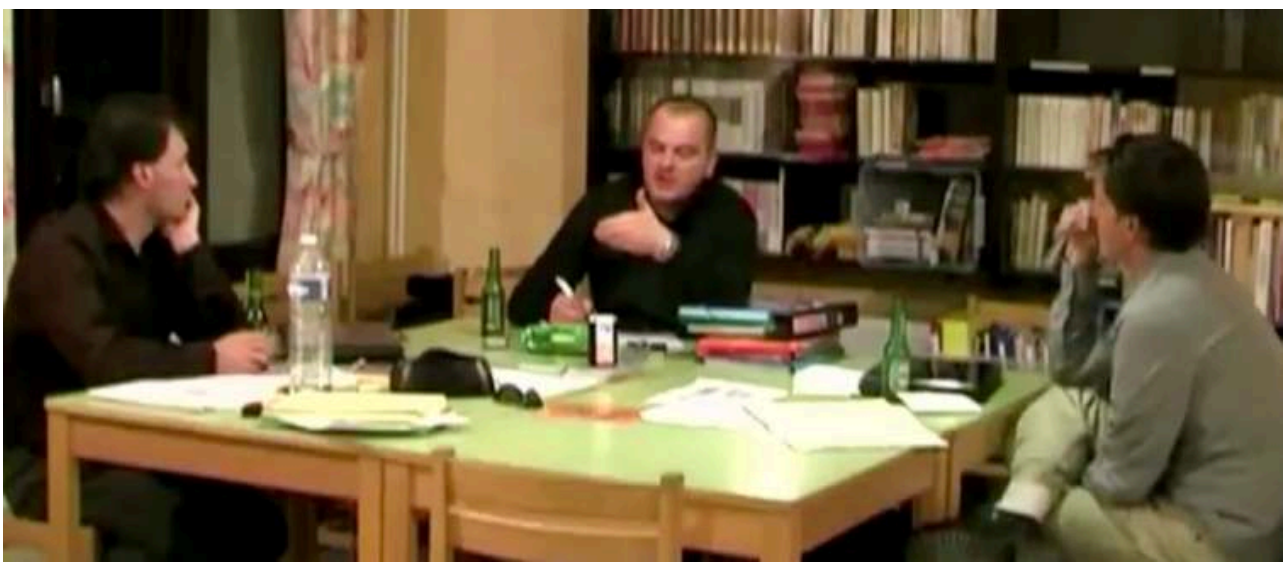

((6 lignes omises))

28 ROG: et j- et avec le TExte/ ça peut être

$29 \rightarrow$ pas mal de faire cet exercice-là $(0.5)$

30 parce que ça fait à la fois une

31 compréhension un peu: d'un texte

32 de loi/

33 (0.7)

34 BER: ouais [ouais]

35 ROG: $\rightarrow$ [et puis] euh:: c'est celui-là

$36(0.8)$

37 BER: ouais c- enfin c'est vraiment celui/

38 que j'ai en tête hein

39 (1.6)

40 ROG: $\rightarrow$ c'est l'arrêté du vingt-deux juin ${ }^{\circ} \mathbf{l a ̀}^{\circ} \#$

fig fig.3\#

Fig. 3

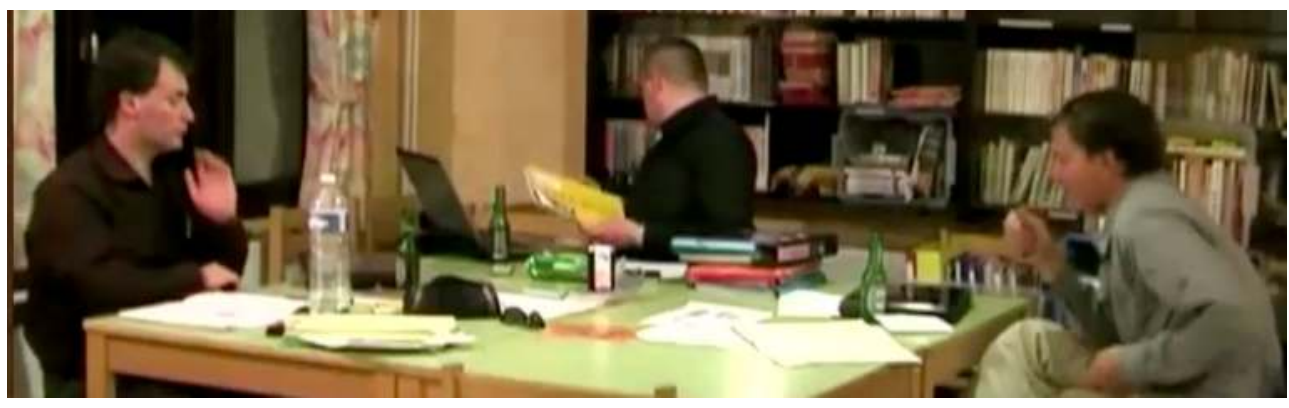

Dans cet extrait, plusieurs occurrences de là sont observables: lignes 8-9 ROG mentionne à nouveau (cf. le début de l'extrait) ce petit texte là et fait un geste cooccurrent avec la forme là (Fig. 1), qui en souligne le caractère spatial et référentiel. Plus loin quand, en réponse à la question de BER, ROG dit je leur ai mis la complète là (14-15) il ne fait en revanche aucun geste (à ce moment-là il est en train de commencer à se tourner vers ses dossiers, Fig. 2). Les occurrences de là avec démonstratif $(23,27)$ ne sont pas non plus accompagnées de gestes. Le dernier là est produit avec une voix faible, donc minimisé vocalement, pendant que ROG est tourné vers ses dossiers : là aussi il ne fait aucun geste. Ainsi on peut remarquer que quand là est spatial (9), il est accompagné d'un geste; quand il va de pair avec un démonstratif (cet exercice.là 23, celui-là 27) le geste n'est pas nécessaire, le là étant désambiguïsé par le démonstratif qui 
le précède ; quand il s'apparente davantage à une particule discursive $(14-15,31)$, il n'est pas accompagné de gestes et est produit pendant que le corps du locuteur est occupé à d'autres mouvements, comme se tourner, chercher des documents, etc. Ainsi, sur la base d'observations préliminaires, il devient possible d'explorer la distinction entre deux catégories, et la grammaticalisation en prenant en compte la gestualité - et plus globalement la multimodalité - comme critère (et surtout ressource pour les participants) supplémentaire permettant de distinguer différentes formes (telles que traitées par les locuteurs).

Plus globalement, ces exemples montrent que le corpus permet d'observer non seulement une pluralité d'emplois de là mais aussi la manière dont les locuteurs traitent cette diversification et, si nécessaire, opèrent des différenciations dans le choix local des ressources utilisées.

\section{Là de structuration}

31 La particule là grammaticalisée en marqueur de structuration occupe deux positions majeures : d'une part elle peut être antéposée, d'autre part elle peut être postposée en fin de clause.

Les cas de là antéposé, en début de clause, sont souvent précédés de connecteurs : parce que là est relevé partout, mais plus fréquemment en France, Nouveau-Brunswick et Congo ; après là est attesté en Côte d'Ivoire, en Belgique, Cameroun et France ; alors que donc là et mais là sont repérables dans toutes les zones, et tout particulièrement en France et en Belgique. De ce point de vue il ne semble pas que ce format puisse différencier substantiellement des zones - même si des quantifications seraient là nécessaires.

Le là postposé, fonctionnant comme particule discursive, a été identifié sous différentes catégories en français - dénommé là d'actualisation (Quéffelec et al., 2997; Ngamountsika, 2012) ou là de clôture (Barberis, 1992). Ces cas de là finaux sont fréquents dans toutes les zones.

Mais leur distribution syntaxique varie.

Dans les français d'Europe, on constate que le là final est souvent précédé d'un syntagme nominal qui peut avoir différentes portées possibles (cf. Barbéris, 1992) :

(11) (FR_PAR_AUT_02)

A: alors vous z- vous m'appelez/ euh

le quatuor là/

(12) (BE_LGG_REP_01)

A: j'ouvre un peu de lasagne là

B: oui

(13) (FR_LIO_REP_03)

$\mathrm{A}:$ je vais prendre euh: les trucs là

Dans ces cas, le là final peut soit clôturer la totalité de la clause, soit le syntagme nominal final, de manière analogue à un format de là répandu dans toutes les aires, le_N_là (voir infra, § 7.).

En revanche, au Canada, il est précédé de constructions beaucoup plus diversifiées, et souvent par des syntagmes verbaux. En voici quelques exemples:

(14) (CA_QC_REP_01)

1 A: ils ont un- au moins ils ont une 


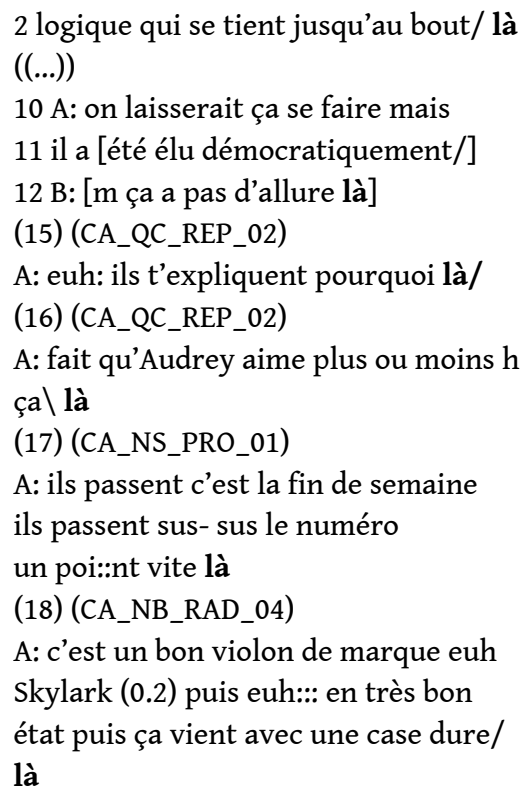

Dans ces cas, le là clôture la clause tout entière et pas uniquement le dernier syntagme. En outre, le sens spatial est estompé - devenant de plus en plus abstrait et métaphorique - pour se grammaticaliser : là indique la complétion du tour.

Ce rôle structurant de là est visible dans un autre type de construction, la relative, qu'il clôt à droite. Ce phénomène a précédemment été décrit à propos de la relative restrictive dans les créoles caribéens (Ludwig \& Pfänder, 2003). Dans le corpus CIEL, on le retrouve dans de nombreux autres aires et notamment au Cameroun. En voici deux exemples :

(19) (CM_YAO_PRO_02)

DAM: on compose du vingt-et-un (0.8)

$<(($ ralenti) $)$ au vingt-quatre mai:/>

(1.5)

DAM: OUI vingt-un vingt-trois mai (0.4)

et puis on a: la semaine qui SUIT là

(20) (CM_YAO_PRO_02)

GAB: oui: au moins// ils ont un-] au moins

ils ont une logique [qui se tient

jusqu'au bout/ là

On remarquera aussi que cette parenthèse droite d'une relative $N+$ qui... là, l'élément sur lequel porte la relative peut être lui-même marqué par là (dans le format le/ mon_N_là) :

(21) (CM_DLA_REP_01)

JOL: après elle vient me suivre elle me

dit que bon: mon gars là qui veut

te voir là/ bon dis à ton gars que:

\section{Rendements interactionnels}

41 Le format le/mon_N_là peut avoir un rendement interactionnel qui a été identifié dans la littérature en termes génériques, en invoquant le marquage d'un common ground : là ferait référence à un élément du savoir partagé et cela lui aurait valu la catégorisation de là « de connivence » (Ludwig \& Pfänder, 2003 : 272). Si cette description renvoie à 
une intuition globale interprétant le là comme pointant vers la mémoire discursive des interlocuteurs, une description interactionnelle permet de mieux en préciser les enjeux, moins en termes cognitifs qu'en termes de pratiques interactionnelles par lesquelles l'intersubjectivité est constamment travaillée et retravaillée par les interlocuteurs (Schegloff, 1992 ; Drew, 1995, Heritage, 2007).

Si l'on se tourne vers des occurrences en interaction, on constate ainsi que le caractère partagé d'un savoir ou d'une référence n'est jamais totalement acquis au fil de l'interaction, mais est constamment vérifié, rappelé, ré-établi, voire établi comme tel lorsqu'il ne va pas de soi pour les interactants (voir p. ex. : Schegloff, 1996 ; Ford \& Fox, 1996, à propos de la référence aux personnes). Cela est visible dans des usages de le_N_là dans des environnements où le locuteur est visiblement engagé dans un travail d'établissement du référent et de son caractère reconnaissable. Ce travail s'implémente dans différentes pratiques.

Cela peut prendre la forme, par exemple, de recherches de mots, dans lesquelles le locuteur cherche et construit graduellement la bonne formulation:

(22) (CM_DLA_REP_01)

A: au fait j'ai mon: (.) .h l'ami de

mon: (.) mon gars là qui veut te voir là

Ici, le locuteur commence la formulation de la personne dont il est question par mon:, dont l'allongement final et la pause successive montrent qu'il y a un problème, suivi non pas du nom projeté mais d'un nouveau syntagme, l'ami de mon:, à nouveau allongé et suivi de pause, finalement suivi de la formulation trouvée, mon gars là, produite sans hésitation et suivie de la continuation du tour.

Cela peut aussi prendre la forme d'une recherche de formulation qui est davantage orientée vers l'autre, vers l'interlocuteur - dont la non-réponse éventuelle peut manifester la non-reconnaissance du référent. En voici un exemple :

(23) (CM_DLA_REP-01)

$1 \mathrm{~A}: \mathrm{j}$ 'ai ma petite copine là

$2(0.4)$

$3 \mathrm{~A}:$ tu connais ma copine là

$4(0.4)$

5 A: la nièce de Depuis là/

6 la plus petite là

$7(0.4)$

8 B: ouais

9 A: hum c'est ma copine là-bas/

10 c'est elle qui

Dans ce cas, A mentionne une première fois ma copine là (1). L'interlocutrice ne répond pas (2). A procède par conséquent en vérifiant explicitement son savoir (tu connais 3 ). Mais B ne répond toujours pas (4). A poursuit en ajoutant des spécifications - toujours sans réponse de l'interlocutrice (6), alors qu'en cette position séquentielle serait attendu un marqueur de changement d'état (comme par exemple ah Heritage, 1984). B ne produit un ouais que plus tardivement (7). Il est significatif que suite à cette réponse, A peut continuer dans la progression de son histoire, en faisant référence cette fois à ma copine là-bas (8) en utilisant un locatif qui est formellement distinct du déictique là (cf. supra). Ici les SN marqués par là sont utilisés dans un environnement où il y a absence de reconnaissance manifestée par le travail de formulation orienté vers cette reconnaissance par le locuteur. 
47 Le locuteur peut aussi avoir recours à ces deux pratiques - recherche de mot et recherche d'une formulation qui correspondent au savoir de l'interlocuteurensemble, comme ici :

(24) (BF_OUA_PRO_01)

$1 \mathrm{~A}$ : TElecel/ tu- tu connais la chargée

2 de communication non/

$3($.

4 B: non/ eh:/ chose- (.) la fille de:

5 Alpha là/

6 (.)

7 A: Alpha là:

8 B: je la connais pas per(so) c'est

9 beaucoup plus Soufo que

10 je connais:/

Dans ce cas, B ne répond pas immédiatement (2) à la question de A mentionnant la chargée de communication (1), tout en s'engageant ensuite dans une recherche du nom de cette personne, accompagnée par là (3). A confirme (5) tout en continuant à s'orienter vers le fait que la référence personnelle n'est pas complètement assurée, comme le montre le là. La réponse finale montre une forme de reconnaissance partielle qui confirme que des problèmes d'identification se posaient précédemment et que les interlocuteurs sont aux prises avec un problème d'établissement graduel de la référence et du savoir partagé.

Ainsi, le tour suivant le là peut manifester soit la non-reconnaissance (comme dans les cas précédents), poussant le locuteur à développer et à spécifier la référence, soit la reconnaissance et donc l'existence d'un savoir partagé, comme dans l'extrait suivant :

(25) (CM_YAO_REP_02)

$1 \mathrm{~A}$ : bon (.) c'est quand même à un niveau:

2 (.) c'est [pas comme les bacheLIERS là

3 B: [oui/ c'est:/ c'est pas comme

4 les bacheliers/

Dans cet extrait, A utilise le format $l e \_N \_l a ̀$. Son interlocuteur, B, montre qu'il s'oriente vers cette référence comme faisant appel à un savoir commun: il manifeste sa reconnaissance, non seulement par le token oui, non seulement en répétant la même forme (sans là, puisque la référence est maintenant établie comme partagée), mais aussi en le faisant très tôt, en chevauchement.

51 De ce point de vue, le placement d'une réponse en oui/ouais après le format le_N_là est récurrent et montre le même phénomène à l'œuvre :

(26) (CI_ABJ_PRO_01)

A: va prendre les parents de les amis-

euh l- de l'apprenti là

B: ouais

(27) (SN_DK_AUT_01)

A: donc/ (.) si j'ai mis/ jau:ne \(0.5)

$\mathrm{j}$ 'ai mis parce que: $\mathrm{y}$ en a plus là-bas $\backslash$

$(2.8)$

A: tu as vu les/ couleurs $\backslash$ (.) là/

B: ouais

(28) (CM_YAO_AUT_04)

$1 \mathrm{~A}$ : dès que je donne la dot là

2 B: ouais

3 A: je leur dis que moi (.) je n'ai 
4 plus $<($ (en riant $))$ l'argent

5 pour le mariage/> montre l'orientation des deux participants vers l'établissement du référent. Dans le dernier exemple il est clair que cela est traité comme une condition pour que le récit puisse progresser (3-4). collaboratifs, comme dans les extraits suivants - l'un enregistré au Cameroun, et l'autre au Canada :

(29) (CM_YAO_AUT_04)

1 SAM: dès que je je je sais sais que

2 [dès que je (.) je donne] la dot là=

3 LOU: [c'est tout ce qu'elle attend]

$4 \mathrm{PAB}:=\mathrm{ils}$ sauront que tu as les moyens

(30) (CA_NB_REP_04)

1 LEO: tu sais ce qui est plus scary

2 dans les films c'est la musique\}

3 SI y avait pas la musique là:

4 KAT: on aurait pas/ ben peur

Dans le premier cas, le caractère partagé de ce dont il est question est visible dans le chevauchement de LOU (3) qui effectue une première complétion collaborative. Mais celle qui nous intéresse est la seconde, par PAB qui enchaîne sur la fin de la ligne 2, exhibant par là que la dot est un référent acquis.

L'observation détaillée de là dans son interactionnel permet ainsi de mieux comprendre plusieurs de ses fonctionnements, tel qu'il est non seulement décrit par le linguiste, mais exhibé par le comportement des locuteurs eux-mêmes.

\section{Conclusion}

Cet article a poursuivi un double objectif : d'une part, présenter le projet CIEL et le corpus qui en a résulté, avec une emphase particulière sur les choix effectués dans sa constitution; d'autre part, proposer un exemple d'analyse exploitant les potentialités comparatives de la base de données, à propos d'une forme polyfonctionnelle du français, là.

L'analyse montre l'importance de pouvoir soumettre aux mêmes interrogations des occurrences d'une forme - mais aussi, dans une perspective plus ample, des constructions, des actions, des types de séquences, des types de formats, etc. - dans différentes aires documentées de manière similaire. L'accès à des données similaires pour des aires communicatives différentes permet non seulement d'explorer des similarités et des différences entre variétés; il permet une forme de symétrie et d'égalité entre ces variétés. Ce dernier point est inédit et permet, par exemple, d'éviter des affirmations telles que 'tel usage n'existe pas dans telle variété' aussi bien que 'tel usage est typique de telle variété', souvent effectuées en absence de corpus comparatifs permettant de resituer la variété étudiée parmi d'autres. Cela permet par là-même de relativiser à la fois l'exotisme' de certaines variétés et la 'familiarité' du français métropolitain : un corpus comparé permet une re-symétrisation et une relativisation des images que les linguistes se font de ces usages. De même, les explorations dans le corpus permettent de nuancer ce qui pourrait être globalement imputé au français 'en 
Afrique' ou 'au Canada' : une base comparée permet de distinguer des tendances entre zones. Ces remarques ouvrent aussi des perspectives et des défis nouveaux, notamment l'intérêt de procéder à des quantifications des occurrences et d'interroger les conditions d'une analyse statistique. Pour cela le corpus CIEL, tout aussi important soitil, peut ne pas être suffisamment vaste, notamment en ce qui concerne des phénomènes moins fréquents. En outre, la question de la quantification ne devrait pas faire oublier l'importance de bien définir à la fois les phénomènes visés et les phénomènes alternatifs observables dans les mêmes environnements - sans quoi la quantification n'a pas de sens (Schegloff, 1993). Cette exigence naît aussi d'une caractéristique majeure du corpus, qui est de mettre à disposition des environnements interactionnels pour l'étude des phénomènes linguistiques, permettant une analyse qui ne se limite pas à la clause ou au discours mais qui intègre fondamentalement les principes de l'interaction sociale. La variété des formes comme des usages naissent des pratiques des locuteurs et de leurs orientations vers des finalités communicatives spécifiques; la langue est constamment façonnée et refaçonnée par eux de manière située et ajustée aux conditions interactionnelles. Cela permet, grâce à des données écologiques, documentant des activités sociales telles qu'elles se déroulent de manière routinière dans leur contexte ordinaire, de penser une linguistique qui réponde à la fois aux défis de la description grammaticale, de la caractérisation sociolinguistique et de l'étude de l'organisation séquentielle et temporelle de l'interaction sociale.

\section{BIBLIOGRAPHIE}

Barbéris J.-M. (1992). « Un emploi déictique propre à l'oral : le là de clôture », in M.A. Morel \& L. Danon-Boileau (éd.) La Deixis. Paris : PUF, 567-578.

Bert M., Bruxelles S., Étienne C., Jouin-Chardon E., Lascar J. \& Mondada L. (2010). « Grands corpus et linguistique outillée pour l'étude du français en interaction (plateforme CLAPI et corpus CIEL) », Pratiques 147-148: 17-34.

Dister A., Gadet F., Ludwig R., Lyche C., Mondada L., Pfänder S., Simon A.C. \& Skattum I. (2008). « Deux nouveaux corpus internationaux du français : CIEL-F (Corpus international et écologique de la langue française) et CFA (Français contemporain en Afrique et dans l'Océan Indien) », Revue de linguistique romane 285/286 : 295-314.

Drew P. (1995). «Conversation analysis : the sequential analysis of intersubjectivity in conversation ", in J. Smith, R. Harré, L. V. Langenhove \& P. Stearns (éd.) Rethinking Psychology. Vol. 2: Alternative Methodologies. London : Sage.

Ehmer O. \& Martinez C. (2014). «Creating a multimodal corpus of spoken world French », in S. Ruhi, M. Haugh, T. Schmidt \& K. Wörner (éd.) Best Practices for Spoken Corpora in Linguistic Research, Newcastle : Cambridge Scholars Publishing, 142-161.

Ford C. E. \& Fox B. A. (1996). «Interactional motivation for reference formulation : he had. This guy had, a beautiful, thirty-two O:lds ", in B. Fox (éd.) Studies in Anaphora. Amsterdam : Benjamins. 
Forget D. (1989). « Là : un marqueur de pertinence discursive », Revue québécoise de linguistique 18 (1) : 57-83.

Gadet F., Ludwig R., \& Pfänder S. (2009). « Francophonie et typologie des situations », Cahiers de linguistique 34 (1) : 143-162.

Gadet F., Ludwig R., Mondada L., Pfänder S. \& Simon A.-C. (2012). « Un grand corpus de français parlé : le CIEL-F Choix épistémologiques et réalisations empiriques ", Revue française de linguistique appliquée XVII/1 : 39-54.

Groupe ICOR (2009). « Exploitation de la plateforme CLAPI : Le cas de voilà dans les chevauchements ", Cahiers de linguistique 33/2:243-268.

Groupe ICOR. (2010). « Grands corpus et linguistique outillée pour l'étude du français en interaction (plateforme CLAPI et corpus CIEL)», Pratiques 147-148 :17-34.

Gumperz, J. (1982). Discourse Strategies. Cambridge : CUP.

Haugen E. (1972). « The ecology of language », in A. S. Dil (éd.) The Ecology of language : Essays by Einar Haugen. Stanford : Stanford University Press, 325-339.

Heritage H. (2007). « Intersubjectivity and progressivity in person (and place) reference », in N. J. Enfield \& S. Levinson (éd.) Person Reference in Interaction : Linguistic, Cultural, and Social Perspectives. Cambridge : Cambridge University Press, 255-280.

Heritage J. C. (1984). « A change-of-state token and aspects of its sequential placement », in J. M. Atkinson \& J. Heritage (éd.) Structures of Social Action. Cambridge : Cambridge University Press, 299-345.

Italia M. (2006). « Le morphème là dans les variétés mésolectales et basilectales en français du Gabon », Le français en Afrique 21 : 281-290.

Levinson S. (1979). « Activity types and language », Linguistics 17 : 365-399.

Ludwig R. \& Pfänder S. (2003). « La particule là/la en français oral et en créole caribéen : grammaticalisation et contact de langues ", in S. Kriegel et al. (éd.) Grammaticalisation et analyse. Approches de la variation créole et française. Paris : Éditions CNRS, 269-284.

Ludwig R., Mühlhäusler P. \& Pagel S. (éd.) (in press). Linguistic Ecology and Language Contact. Cambridge : Cambridge University Press.

Mondada L. (2007). « Multimodal resources for turn-taking: Pointing and the emergence of possible next speakers », Discourse Studies 9, 2 : 195-226.

Mondada L. (2011). «Exigences analytiques pour l'enregistrement de la parole-en-interaction », Version 3.0.2. http://cielf.org/assets/files/Enregistrer_protocole_Mondada.pdf (consulté le 18.2.2015).

Mondada L. (2012). «The conversation analytic approach to data collection ", in J. Sidnell \& T. Stivers (éd.) Handbook of Conversation Analysis. Oxford : Blackwell-Wiley.

Mondada L. (2013). « Displaying, contesting, and negotiating epistemic authorities in social interaction », Discourse Studies 15 : 597-626.

Mondada L. (à paraître). « Conversation analysis », in E. Weigand (éd.) Language and Dialogue : A Handbook of Key Issues in the Field.

Ngamountsika, E. (2012). « Analyse morphosyntaxique du morphème là en français parlé en République du Congo », Revue du français en Afrique, 189-199. 
Ochs E. (1979). « Transcription as theory », in E. Ochs \& B. Schiefflin (éd.) Developmmental Pragmatics. New York : Academic Press.

Pfänder S. \& Skrovec M. (2011). « Donc, entre grammaire et discours. Pour une reprise de la recherche sur les universaux de la langue parlée à partir de nouveaux corpus ", in M. Drescher \& I. Neumann-Holzschuh (éd.) Syntaxe de l'oral dans les variétés non hexagonales du français. Tübingen : Stauffenburg Verlag.

Ploog K. (2006). « Du continuum pragmatico-sémantique aux caractéristiques prosodiques de là en français abidjanais ", Le français en Afrique 21 : 303-323.

Queffélec A. et al. (1997). Le français en Centrafrique : Lexique et société. Paris : Vanves-Edicef. Sacks H. (1972). « An initial investigation of the usability of conversational materials for doing sociology », in D. Sudnow (éd.) Studies in Social Interaction. New York : Free Press, 31-74.

Schegloff E. A. (1992). « Repair after next turn : the last structurally provided for place for the defence of intersubjectivity in conversation », American Journal of Sociology 95 (5) : 1295-1345.

Schegloff E. A. (1993). « Reflections on quantification in the study of conversation », Research on Language and Social Interaction 26 (1) : 99-128.

Schegloff E. A. (1996). « Some practices for referring to persons in talk-in-interaction : a partial sketch of a systematics », in B. Fox (éd.) Studies in Anaphora. Amsterdam : Benjamins, 437-485.

Simon A. C., Gadet F., Ludwig R., Mondada L., Pfänder S. \& Skrovec, M. (2012). Conventions de transcription CIEL-F, Version 4.7. http://ciel-f.org/assets/files/conventions_ciel-f.pdf (consulté le 18.2.2015).

Skrovec M. \& Pfänder S. (2012). « Rhétorique ordinaire et tâches communicatives : convergences et divergences dans des entretiens médiatisés d'experts africains et français », Le français en Afrique, 93-107.

Vincent D. (1981). « C'est ici ou là ? C'est ici là », in D. Sankoff \& H. Cedergren (dir.) Variation Omnibus. Edmonton : Linguistic Research Inc., 437-444.

Wiesmath R. (2003). « La particule là dans le parler acadien du Nouveau-Brunswick / Canada », in S. Kriegel (éd.) Grammaticalisation et réanalyse. Approches de la variation créole et française. Paris : Éditions CNRS, 284-302.

\section{NOTES}

1. http://www.projet-pfc.net

2. http://www.lll.cnrs.fr/eslo-1

3. http://clapi.ish-lyon.cnrs.fr

4. http://www.uclouvain.be/81834.html

5. http://www.ciel-f.org

6. Co-financement par l'ANR en France (ANR-08-FASHS-004) et la DFG en Allemagne (2009-2012 ; Pf 699/1-1; Lu 529/3-1).

7. http://clapi.ish-lyon.cnrs.fr

8. http://moca.phil2.uni-freiburg.de et http://www.uclouvain.be/260466.html

9. Les collaborateurs sont mentionnés dans le site du projet: http://www.ciel-f.org/qui. Sans eux le corpus dans sa richesse et sa diversité n'aurait pas pu voir le jour.

10. http://www.fon.hum.uva.nl/praat/

11. Ces chiffres renvoient à l'année 2014, voir http://clapi.ish-lyon.cnrs.fr consulté le 18.2.2015. 
12. Voir http://moca.phil2.uni-freiburg.de.

\section{RÉSUMÉS}

Cet article présente le travail de constitution du Corpus International Écologique de la Langue Française (CIEL-F) et ses caractéristiques. Conçu pour mettre à disposition des corpus de données interactionnelles récoltées dans des contextes ordinaires, professionnels et institutionnels authentiques, et afin de promouvoir la recherche comparée sur le français parlé, le corpus CIEL-F comporte des enregistrements effectués en Algérie, Antilles françaises, Belgique, Burkina Faso, Cameroun, Canada, Congo, Côte d'Ivoire, Egypte, France, Inde, La Réunion, Maurice, Sénégal, Suisse et Togo. Dans la première partie, l'article présente les défis et les enjeux de ce type de corpus. Dans la deuxième partie, l'article offre un exemple d'exploitation de ces données, en se penchant sur différents usages de là, allant de l'emploi déictique locatif à des emplois qui relèvent davantage de la particule discursive grammaticalisée. L'analyse propose quelques remarques sur la distribution de ces emplois de là dans différentes aires communicatives et des réflexions sur les possibilités ouvertes par une approche comparative au sein du français parlé dans le monde.

This paper describes the conception and constitution of the International Ecological Corpus of French (Corpus International Écologique de la Langue Française CIEL-F). This corpus has been gathered and set up in a data bank in order to make available to the scientific community interactional data collected in ordinary, professional and institutional contexts. The aim is to make possible and promote comparative analysis of spoken French. Data were recorded in Algeria, the French Antillean islands, Belgium, Burkina Faso, Cameroun, Canada, Congo, Ivory Cost, Egypt, France, India, La Réunion, Maurice, Senegal, Switzerland and Togo. In the first part of the article, we present the issues and challenges of such a corpus. In the second part of the article, we offer an example of exploitation of the data bank. More particularly, we study some usages of the form là, which can be used as a locative deictic but also in more metaphorical way as well as a fully grammaticalized discourse particle. The analysis shows the way different usages are distributed in various communicative areas and proposes some thoughts about possibilities and limitations of comparative analyses of French as it is talked across the world.

\section{INDEX}

Mots-clés : français parlé, corpus, enregistrement, écologie, interaction sociale, analyse comparée, là, deixis, particule discursive, grammaticalisation

Keywords : spoken french, corpus, recording, ecology, social interaction, comparative analysis, là, deixis, discourse particle, grammaticalization

\section{AUTEURS}

\section{LORENZA MONDADA}

UMR ICAR Lyon \& Université de Bâle 


\section{STEFAN PFÄNDER}

Université de Freiburg-im-Breisgau 